\section{Yeast solar panels}

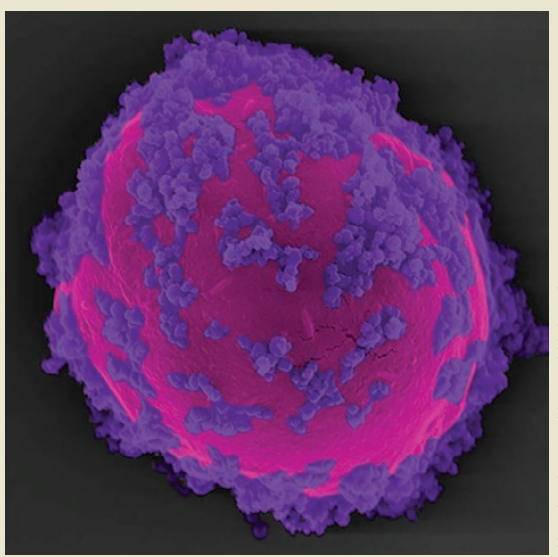

Semiconductor nanoparticles (violet) attached to the surface of a yeast cell (magenta) capture electrons from light to boost production of shikimic acid, a precursor to Tamiflu. Microbes, especially bacteria and yeast, have long been used as biofoundries to produce drugs and fine chemicals at industrial scale. Now researchers have combined yeast with semiconductor technology to harvest energy from light to fuel their biosynthetic pathways. Such a biological-inorganic hybrid was developed by a team from Harvard's Wyss Institute for Biologically Inspired Engineering (Science $\mathbf{3 6 2}$, 813-816, 2018). Like solar panels on the roof of a house, when illuminated, the semiconductor nanoparticles capture electrons and hand them over to the surface of the baker's yeast Saccharomyces cerevisiae, where they energize NADPH molecules, which can fuel reductive biosynthetic reactions. This cost-effective living system creates a scalable blueprint for future biohybrid technologies in which the identity of the semiconductor and the engineered cell can be varied in a plugand-play fashion to manufacture a wide range of medicines and chemical products.

\section{CORRECTIONS}

In the December 2018 issue, the News Analysis "Radioactive drugs emerge from the shadows to storm the market" reversed the descriptions of Actinium Pharmaceuticals' lomab-B and Nordic Nanovector's 177Lu-lilotomab satetraxetan (Betalutin). lomab-B uses iodine-131 to destroy the bone marrow ahead of stem cell transplantation and Betalutin directs lutetium-177 to CD45-expressing B cells in follicular lymphoma.

In the October 2018 issue, the News Feature "The virome hunters" described the work of the PREDICT project incorrectly, and the associated citation was incorrect. The text that read "PREDICT scientists have sampled more than 70,000 animals and people in over 30 countries with high zoonotic disease risks, and the researchers have reported on the discovery of 1,000 virus species with the potential to infect human beings" 5 " has been changed to "PREDICT scientists have sampled more than 70,000 animals and people in over 30 countries at high-risk interfaces and, using consensus PCR, have discovered close to 1,000 new virus species. In bats, for example, which are now believed to be the evolutionary source of viruses such as SARS and MERS, the group detected sequences from 91 coronaviruses in 20 countries 5 ." Ref. 5 has been changed from Olival, K.J. et al. Nature 546, 646-650 (2017) to Anthony, S.J. et al. Virus Evol. 3, vex012 (2017). 